\title{
Luminescence of Gold Nanorod-Quantum Dots Complexes
}

\author{
L. L. Trotsiuk*, A. O. Muravitskaya, O. S. Kulakovich \\ and S. V. Gaponenko \\ B. I. Stepanov Institute of Physics NASB \\ Nezavisimosti Ave. 68-2, 220072 Minsk, Belarus \\ *l.trotsiuk@ifanbel.bas-net.by \\ H. V. Demir \\ UNAM-Institute of Materials Science and Nanotechnology \\ Bilkent University, 06800 Ankara, Turkey
}

Received 22 December 2018

Accepted 5 January 2019

Published 13 May 2019

\begin{abstract}
The photoluminescence of gold nanorod-quantum dots complexes was investigated in order to find optimal conditions for the luminescence enhancing. The number of quantum dots and polyelectrolyte layers on the gold nanorod surface in these complexes were considered as main factors in the enhancement.
\end{abstract}

Keywords: Luminescence; enhancement; gold nanorods; quantum dots.

\section{Introduction}

Quantum dots (QDs) have unique optical properties due to the quantum confinement effect, that determine their wide application in different areas particularly in producing LEDs, solar cells, TV displays as well as biomedical diagnostics. ${ }^{1}$

Plasmon-enhanced luminescence (PEL) allows increasing the sensitivity of diagnostic methods and improving the luminescent characteristics of different optical devices based on quantum dots. PEL could be a perfect tool to expand opportunities of QD applications. PEL is a result of an increase in the number of radiative transitions in the presence of plasmon nanoparticles. However, nearby plasmon nanoparticle, the probability of not only radiative transitions increases but also nonradiative ones, that can lead to the luminescence quenching. Thereby, there are some problems that make it difficult to use PEL in practical applications.

An enhancement of luminescence is sensitive to many parameters like the distance between the plasmon nanoparticle and an analyte, the environment of nanoparticle, and the position of plasmon band relative to the extinction and luminescence bands of the analyte. ${ }^{2}$ In this way, gold nanorods (GNRs) have an advantage over spherical nanoparticles due to the presence of two plasmon bands. ${ }^{3}$

The aim of this work was to study CdSe/CdZnS quantum dot photoluminescence in complexes with gold nanorods in order to find conditions of the plasmon enhanced photoluminescence. We investigated the effect of spacer thickness on gold nanorods as well as the amount of QDs in such complexes on the photoluminescence intensity. 


\section{Experimental Method}

\subsection{Synthesis of gold nanorods}

The GNRs were synthesized according to the seedmediated growth method. ${ }^{3}$ First, the seed solution was prepared by mixing $0.5 \mathrm{mM} \mathrm{HAuCl}_{4}$ solution with $0.1 \mathrm{M}$ CTAB solution and $0.2 \mathrm{M}$ ice-cold solution of $\mathrm{NaBH}_{4}$. Next, the growth solution was prepared mixing $0.1 \mathrm{M} \mathrm{CTAB}, 4 \mathrm{mM} \mathrm{AgNO} \mathrm{An}_{3}$ and $1 \mathrm{mM}$ $\mathrm{HAuCl}_{4}$. The subsequent addition of ascorbic acid $(0.1 \mathrm{M})$ resulted in discoloration of the bright orange mixture. Finally, $150 \mu \mathrm{l}$ of the seed solution was added to the growth solution.

\subsection{Formation of gold nanorod-quantum dots electrostatic complexes}

GNRs were coated with 2, 4 and 6 polyelectrolyte $(\mathrm{PE})$ layers via layer-by-layer technique ${ }^{4}$ using $10 \mathrm{mg} / \mathrm{mL}$ solutions of poly(sodium 4-styrenesulfonate) (PSS) and poly(diallyldimethylammonium chloride) (PDADMAC) in $10 \mathrm{mM} \mathrm{NaCl}$ solution. $1 \mathrm{~mL}$ of GNR solution was added first to $300 \mu \mathrm{L}$ of negative charged PSS solution. After $30 \mathrm{~min}$, this mixture was twice centrifuged, redispersed in deionized water and added to $300 \mu \mathrm{L}$ of PDADMAC solution. The same manipulations were repeated for subsequent deposition of next polyelectrolyte layers.

GNRs coated with $0,2,4$ and 6 polyelectrolyte layers were diluted to the concentration $1 \times$ $10^{-10}$ M. Further, aqueous solution of CdSe/CdZnS quantum dots stabilized with thioglycolic acid was added to GNRs solutions in certain amounts to achieve QD/GNR ratio of $2.5,5,7,10,12,27$ and 51 . The pure quantum dot solutions of the same concentrations were used as reference solutions.

Fluorescence was studied under excitation by 532-nm $c w$ Nd-laser.

\section{Results and Discussion}

Original GNRs were characterized by two plasmon resonances: transversal at $520 \mathrm{~nm}$ and longitudinal at $615 \mathrm{~nm}$. After covering them with PE layers the resonances were slightly shifted [Fig. 1(a)] into the red region because of the changes in local refractive index around GNR. The luminescence band of QDs with the maximum at $620 \mathrm{~nm}$ [Fig. 1(b)] coincided with the longitudinal and the excitation wavelength at $532 \mathrm{~nm}$ was overlapped with the transversal resonance of GNRs and absorption band of QDs.

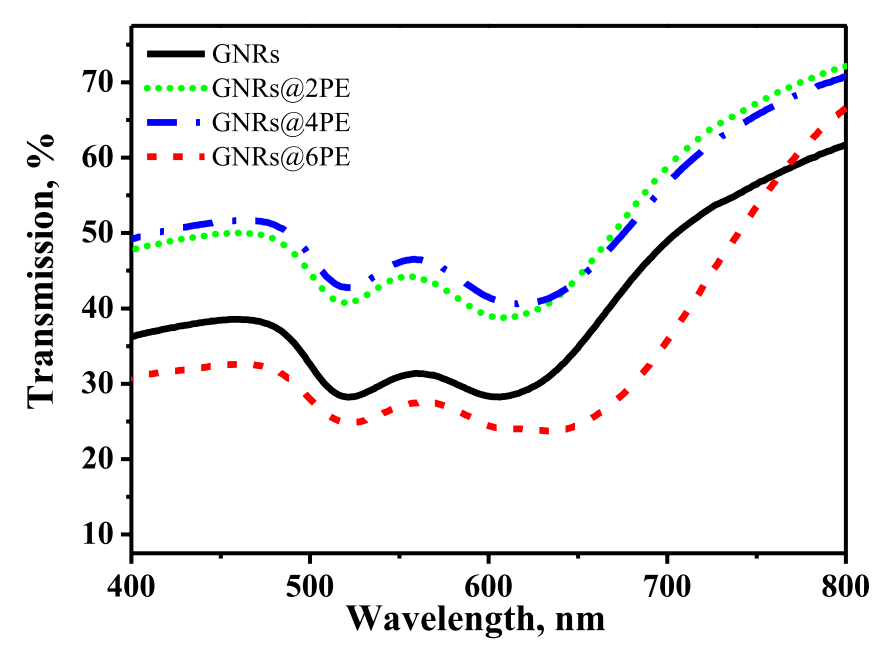

(a)

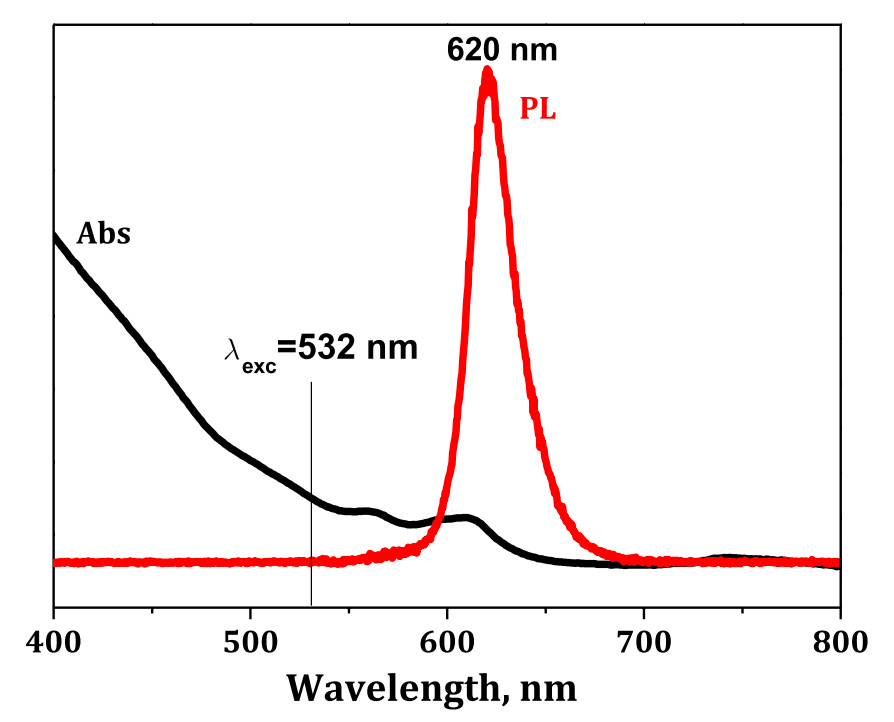

(b)

Fig. 1. (a) Transmission spectra of original GNRs and GNRs covered with 2, 4 and 6 polyelectrolyte layers. (b) Absorption and luminescence spectra of QDs.

Many experimental works showed the optimal distance to obtain the luminescence enhancement must be $10-20 \mathrm{~nm}^{5-7}$ However, according to the theoretical calculation, ${ }^{7}$ the strong luminescence enhancement on the GNR can be observed at small distances $(3-5 \mathrm{~nm})$ in the case of the emitter localization on the end of the rod and at the excitation of the longitudinal plasmon resonance. The excitation of transversal resonance or emitter localization on the sides of the nanorod lead to the quenching. Therefore, we investigated the effect of QD concentration in complexes with GNR covered with 2 PE. Due to the CTAB bilayer on the GNR surface, which thickness is about $3.2 \mathrm{~nm}$, the distance between the GNR and QD in these complexes is about $5 \mathrm{~nm}$. 


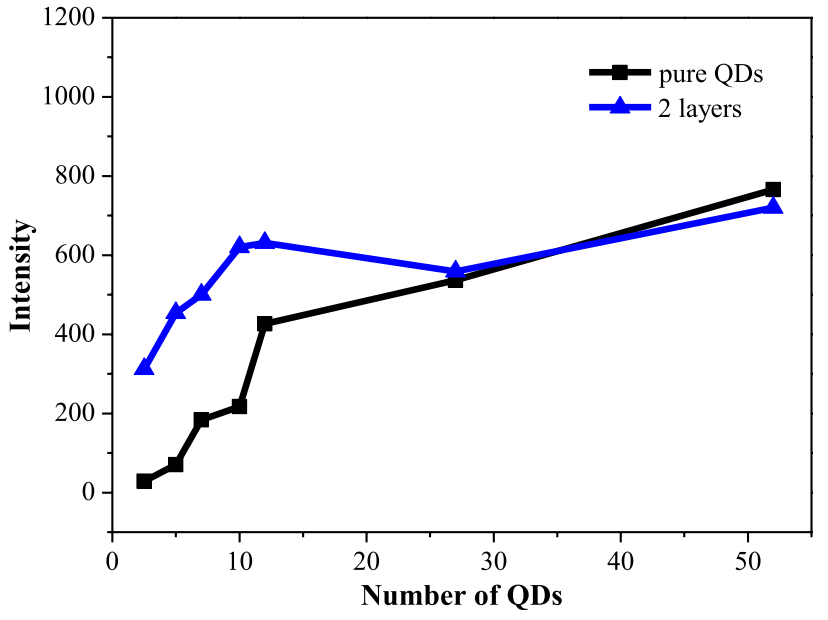

Fig. 2. Luminescence measurements of pure QD solution and GNR-QDs complexes with 2PE layers as a dielectric shell.

Table 1. Enhancement factor (EF) for GNR-QD complexes with 2 PE layers as a dielectric shell.

\begin{tabular}{lccccccc}
\hline QD/GNR ratio & 2.5 & 5 & 7 & 10 & 12 & 27 & 52 \\
\hline $\mathrm{EF}$ & 10.8 & 6.4 & 2.7 & 2.9 & 1.5 & 1 & 0.9 \\
\hline
\end{tabular}

In this work, we observed strong dependence of the luminescence intensity on the number of QDs per rod in complexes (Fig. 2). At first, the increase of $\mathrm{QD}$ amount led to the growth of the luminescence intensity, but after achieving 12 QDs per rod, some saturation appeared. As a result the decrease in the luminescence intensity appeared. It, probably, indicates that only about 12 QDs can be deposited on the GNR surface without their interaction and self-quenching due to the Förster resonance energy transfer.

Table 1 shows the enhancement factor $(\mathrm{EF})$ of the fabricated complexes. The maximal EF was obtained in the complex with 2.5 QDs per rod. Then, it falls fast with the increase in QD number. Probably, in the case of 2.5-5 QDs per rod, QDs were localized on the opposite ends of the rod that was an optimal position for the luminescence enhancing. Further growth of QD amount led to fast decrease in EF. Probably, it is due to the deposition of QDs on the sides of the rod. In this case, EF was the sum of the strong luminescence enhancing on the ends of the rod and the quenching on its sides.

We also studied the effect of the distance in complexes with the optimal number of QDs (2.5 QDs per rod). Figure 3 shows the enhancement factor in GNRQDs complexes with different numbers of PE layers and consequently the distance between them.

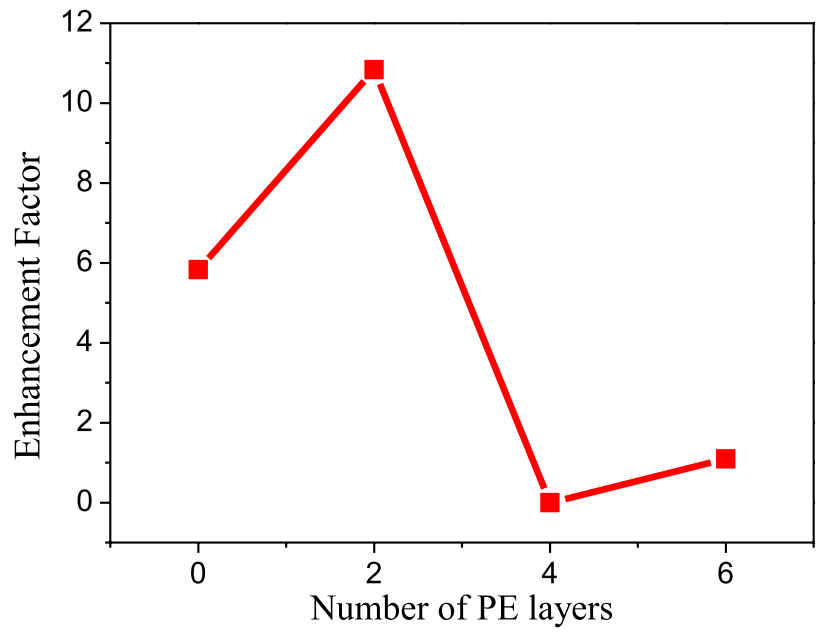

Fig. 3. The enhancement factor of luminescence as a function of the number of PE layers in the GNR-QD complexes.

The results are in good agreement with theoretical calculations, ${ }^{8}$ that predicted the maximal enhancement at the distances of $3-5 \mathrm{~nm}$, because of the high probability of the radiative transitions. It is intriguing, that small increase in the distance (from about 5 to $7 \mathrm{~nm}$ for 2 and $4 \mathrm{PE}$ layers) led to the strong falling of the EF.

\section{Conclusion}

We created GNR-QDs complexes and demonstrated the optimal conditions for an enhancement of their luminescence. The number of QDs and the distance between GNR and QDs play an important role for a domination of radiative transitions over nonradiative. The maximal EF of 10.8 was observed in the complexes with 2.5 QDs per GNR and with 2 $\mathrm{PE}$ layers as a dielectric spacer between them.

\section{References}

1. M. V. Kovalenko et al., ACS Nano 9, 1012 (2015).

2. S. V. Gaponenko, Introduction to Nanophotonics (Cambridge University Press, 2010).

3. B. Nikoobakht and M. A. El-Sayed, Chem. Mater. 15, 1957 (2003).

4. F. Caruso, Adv. Mater. 13, 11 (2001).

5. N. S. Abadeer, M. R. Brennan, W. L. Wilson and C. J. Murphy, ACS Nano 8, 8392 (2014).

6. F. Tam, G. P. Goodrich, B. R. Johnson and N. J. Halas, Nano Lett. 7, 496 (2007).

7. O. Kulakovich, N. Strekal, A. Yaroshevich, S. Maskevich, S. Gaponenko, I. Nabiev, U. Woggon and M. Artemyev, Nano Lett. 2, 1449 (2002).

8. G. Lu, T. Zhang, W. Li, L. Hou, J. Liu and Q. Gong, J. Phys. Chem. C 115, 15822 (2011). 